\title{
Cross Section TEM Characterization of High-Energy-Xe-Irradiated U-Mo
}

\section{B. Yea, , L. Jamisona, Y. Miaoa, S. Bhattacharyab, G.L. Hofmana, A.M. Yacouta}

aNuclear Engineering Division, Argonne National Laboratory, 9700 S. Cass Ave. Lemont, IL 60439, USA

bDepartment of Materials Science and Engineering, Northwestern University, 2220 Campus Dr.

Evanston, IL 60208, USA

${ }^{*}$ Corresponding author

Tel: +1 630252 4506;

Fax: +1 6302525161 ;

E-mail address: bye@anl.gov.

Submitted for publication in Journal of Nuclear Materials

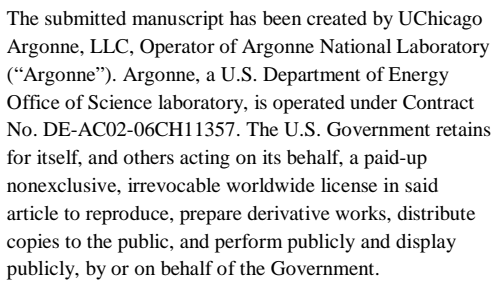

(C) 2016. This manuscript version is made available under the Elsevier user license http://www.elsevier.com/open-access/userlicense/1.0/ 


\begin{abstract}
U-Mo alloys irradiated with $84 \mathrm{MeV}$ Xe ions to various doses were characterized with transmission electron microscopy (TEM) and scanning transmission electron microscopy (STEM) techniques. The TEM thin foils were prepared perpendicular to the irradiated surface to allow a direct observation of the entire region modified by ions. Therefore, depth-selective microstructural information was revealed. Varied irradiation-induced phenomena such as gas bubble formation, phase reversal, and recrystallization were observed at different ion penetration depths in UMo.
\end{abstract}

Key words: heavy ion irradiation, nuclear fuel, fission gas bubble, recrystallization 


\section{Introduction}

U-Mo alloys (with 7-10 wt.\% Mo) with a low U-235 enrichment ( $<20 \%$ ) have been developed to be used in high-performance research and test reactors, replacing the current highly enriched uranium (HEU) fuels [1]. A number of in-pile irradiation tests of U-Mo fuels [2]-[5] show that drastic microstructural changes occurred in U-Mo during reactor irradiation, including formation of a high density of fission gas bubbles and irradiation-induced recrystallization. Accumulation of fission gas bubbles is the main cause of total fuel swelling [6], and the recrystallization process may lead to the observed accelerated fuel swelling at high burnup [7]. Because fuel performance is closely related to the microstructural evolution during irradiation, many efforts have been made aiming to understand the underlying mechanisms of these microstructural changes. Heavy ion irradiation has been employed as a useful tool to investigate separate effects of the irradiation behavior of U-Mo fuels [8]-[11]. Highenergy iodine or xenon ions were implanted into U-Mo/Al samples to simulate irradiation damage generated by fission fragment bombardment. Most of the results reported from these studies focused on the irradiation-induced interdiffusion behavior between U-Mo and $\mathrm{Al}$, an important factor limiting U-Mo/Al dispersion fuel performance [12]. For example Chiang et al. described the evidence of amorphous interdiffusion layer observed in heavy ion irradiated U-8wt\%Mo/Al interfaces in ref. [10]. Detailed information of the depth distribution of the microstructural features in U-Mo has not been described. The defect microstructure as a function of irradiation damage is imperative to gain a deep understanding of the radiation effects in nuclear fuels in reactors. Particularly, this information is of great interest for developing theoretical models to describe the microstructure evolution process in U-Mo fuels.

This study used transmission electron microscopy (TEM) to investigate microstructural effects in Xe-ionimplanted U-7wt\%Mo (denoted as U-7Mo) alloy. The same method was applied to study the depth profile of damage accumulation in heavy-ion-irradiated $\mathrm{UO}_{2}$ fuels [13]. In addition to the TEM characterizations, the irradiated materials were also characterized using synchrotron radiation X-rays. The results are consistent with this study and will be summarized separately. The irradiation details were described in a previous paper [11]. Figure 1 outlines the sample configuration and preparation procedure before irradiation. Figure 1 (a) and (b) show that a 1.7mm-diameter disk is punched out from a U-Mo/Al dispersion fuel plate. The fueled zone is composed of U-7Mo fuel particles in an $\mathrm{Al}$ matrix. Before irradiation, one side of the Al cladding was polished away to expose the fueled zone, as illustrated in Figure 1(c). Multiple samples in the same configuration were irradiated with a defocused Gaussian Xe beam at the ATLAS accelerator at Argonne National Laboratory (ANL). The ion source was 84-MeV 
Xe ions that was selected for two reasons: (1) to simulate the irradiation response of the fuel to fission fragment bombardments, and (2) to study fission gas behavior, as fission gas bubble formation and growth is the primary cause of U-Mo fuel swelling [6][7]. The final doses achieved in the irradiation varied in a range of $1.8-2.9 \times 10^{17}$ ions $/ \mathrm{cm}^{2}$, depending on sample location. The ion-irradiated samples were first characterized with scanning electron microscopy (SEM); the results have been reported earlier [11]. However, the resolution of the SEM technique was not high enough to reveal the sub-micron damage structures in the irradiated material. In this work, detailed TEM/STEM (scanning transmission electron microscopy) was, therefore, performed to obtain structural and chemical information of heavy-ion-irradiated U-Mo on sample cross sections at nano and atomic scale.

\section{Experimental details}

After irradiation, TEM lamellas were prepared using the focused ion beam (FIB) technique from samples irradiated to a dose of $1.8 \times 10^{17}, 2.6 \times 10^{17}$, and $2.9 \times 10^{17}$ ions $/ \mathrm{cm}^{2}$ respectively. Table 1 provides the irradiation parameters of the TEM samples analyzed in this work. FIB is the most viable way to prepare samples for crosssection TEM observation in this work, because it allows precise selection of interested region which is only a few microns below the sample surface. The Xe ion distribution in U-7Mo, shown in Fig. 2, was calculated with SRIM2008 [14] using the "ion distribution and quick calculation of damage" option with a displacement energy of $50 \mathrm{eV}$ and $25 \mathrm{eV}$ for $\mathrm{U}$ and Mo, respectively. According to the calculation, the $84 \mathrm{MeV}$ Xe ions have a penetration depth of $\sim 6 \mu \mathrm{m}$, with peak deposition occurring $4.5 \mu \mathrm{m}$ away from the surface. In order to encompass the entire interested range, TEM samples were created at least $10 \mu \mathrm{m}$ in depth from the sample surface. The TEM samples were lifted out from the center of U-Mo particles to avoid the Al matrix. TEM characterization was carried out using either a Hitachi-9000 system at $300 \mathrm{kV}$ at ANL or a 300-kV FEI Tecnai TF30-FEG STEM at Center for Advanced Energy Studies (CAES).

\section{Results}

\subsection{U-Mo microstructure features along the ion penetration depth in the sample irradiated to $2.9 \times 10^{17}$ ions $/ \mathbf{c m}^{2}$}

A montage of TEM micrographs in Figure 3 shows representative cross-section microstructures of the sample irradiated to $2.9 \times 10^{17}$ ions $/ \mathrm{cm}^{2}$. From characteristic features, the sample can be divided into 3 zones along the ion penetration direction, starting from the surface: 
1) Zone $\mathrm{A}$ (depth is $0-1.3 \mu \mathrm{m})$ is the $(\mathrm{U}, \mathrm{Mo}) \mathrm{Al}_{\mathrm{x}}$ layer formed due to the reaction between $\mathrm{U}-\mathrm{Mo}$ and the $\mathrm{Al}$ sputtered onto the U-Mo surface during irradiation;

2) Zone B (depth is 1.3 - $4.7 \mu \mathrm{m})$ consists solely of Xe-implanted U-Mo;

3) Zone C comprises the U-Mo beyond the ion penetration depth.

The detailed microstructure of the area marked with "A" in Fig. 3 is presented in Fig. 4 (a), from which the grain size of the polycrystalline reaction product is estimated to be $50-200 \mathrm{~nm}$. The composition of the reaction product in zone $A$ is $(\mathrm{U}, \mathrm{Mo}) \mathrm{Al}_{\mathrm{x}}$ with an $\mathrm{x}$ value ranging from $3.5-7.3$ [11]. In order to identify the phase composition of the reaction product, the intensity variation of the diffraction rings across the diffraction pattern (inset) taken from the same area was plotted in Fig. 4 (b) with ImageJ [15]. The indexing results show that the major phases existing in the area are $\mathrm{UAl}_{3}$ and $\mathrm{UAl}_{4}$, consistent with X-ray powder diffraction (XRD) measurement results on U-Mo/Al dispersion fuels irradiated with high-energy iodine ions [8].

Large gas bubbles (10 - $150 \mathrm{~nm}$ in diameter) were seen in zone B in Fig. 3. These bubbles formed in the region that is $\sim 4-5.5 \mu \mathrm{m}$ from the surface. The location of the large bubbles corresponds with the region of greatest Xe ion deposition estimated with SRIM (Fig. 2). Both the size and the linear arrangement of the large bubbles suggest that they are intergranular bubbles. The facts that the grains delineated by these SEM-observable bubbles are much smaller than the original cell structures (sub-micron vs. a few microns) and were only observed in irradiated areas signify that these are new grains formed through irradiation-induced recrystallization.

In addition to the bubbles on grain boundaries, high-density bubbles in a size of a few nanometers exist within fuel grains in zone B. A much higher magnification view of the marked region "B" is provided in Fig. 5. Figs. 5 (a) and (b) are a set of bright-field TEM images taken at over-focus and under-focus conditions, respectively. The opposite contrast of the high-density nano-size features shown in Figs. 5 (a) and (b) demonstrates that they are gas bubbles [16]. For the purpose of quantitative analysis, high angle annular dark field (HAADF) scanning transmission electron microscopy (STEM) images taken at various depths were used for size measurement, because the contrast of the gas bubbles compared to its surrounding area is much stronger, making it more suitable for quantitative measurements than conventional TEM images. The density and average size of the intra-granular bubbles as a function of distance from the surface is depicted in Fig. 6. For each data point of bubble size in Fig. 6, at least 600 gas bubbles were sampled. Local sample thickness was measured with electron energy loss spectroscopy (EELS). Bubble volume density can therefore be estimated using areal density and measured thickness. 
No gas bubbles were found less than $3 \mu \mathrm{m}$ distance from the surface. SRIM calculation predicts very low gas deposition in this range, which is too low to form gas bubbles. Most of the gas atoms deposited in this range dissolved in the U-Mo lattice. The measurement data show that the variation of average intra-granular bubble size is small across the bubble formation region ( $3-5.5 \mu \mathrm{m}$ from the surface). The average bubble size is $\sim 4.5 \mathrm{~nm}$ in diameter, close to the reported intra-granular bubble size in the in-pile-irradiated U-Mo fuels [17]-[19]. The intragranular bubble density shows a slight increase at a distance $3-4 \mu \mathrm{m}$ from the surface and a decline $\sim 5 \mu \mathrm{m}$ from the surface. The increase of gas bubble density corresponds to the increase of deposited gas concentration, and the reduced bubble density and slightly larger bubble sizes near the peak position of Xe ion concentration is due to the coalescence of the intra-granular bubbles.

Fig. 7 (a) shows the representative microstructure of zone C, which is located beyond the Xe ion stopping range. No Xe bubbles were found in this region. Precipitation in a somewhat poorly defined Widmanstätten form was observed. These precipitates are in a size up to $100 \mathrm{~nm}$ and show a certain orientation relationship with the $\gamma$ uranium matrix. The precipitates were only found in zone $\mathrm{C}$ and not in zone B. Furthermore, their density increases with distance from the sample surface. The selected area diffraction (SAD) pattern in Fig. 7 (b) shows that in addition to the $\gamma-\mathrm{U}$ matrix (the main diffraction spots), a certain amount of secondary-phase material exists in the sample. Indexing of the SAD pattern reveals that the ring pattern belongs to $\mathrm{UO}_{2}$, which likely formed during sample preparation and storage, and the weak diffraction spots are from $\alpha-\mathrm{U}$ precipitates. Fig. 7 (c) shows a TEM dark-field image using the diffraction spots marked in Fig. 7 (b). The $\alpha-U$ precipitates in one particular orientation show bright contrast.

In order to verify whether the $\alpha-U$ precipitates formed during ion irradiation, TEM examination was also performed on a reference sample, which was prepared from the same plate as the specimen shown in Fig.3. The bright-field (BF) TEM micrograph (Fig. 8 (a)) clearly shows that dense secondary-phase precipitates exist near cell boundaries in the unirradiated sample, and the scattered weak diffraction spots in Fig. 8 (b) also confirm those secondary-phase precipitates are $\alpha$-phase U. The cellular structure in U-Mo particles formed during the atomization process of U-Mo particles due to rapid solidification of the melt U-Mo [20] - [24].

\subsection{Microstructure of U-Mo irradiated at lower doses}

TEM specimens prepared from samples irradiated to lower doses were examined as well. Low-magnification overviews of the samples irradiated to $2.6 \times 10^{17} \mathrm{ions} / \mathrm{cm}^{2}$ and $1.8 \times 10^{17}$ ions $/ \mathrm{cm}^{2}$ are shown in Fig. 9 (a) and (c) 
respectively, and their magnified views are presented in Fig. 9 (b) and (d). For the purpose of comparison, images taken at the same magnification were arranged side by side in Fig. 9. These micrographs were taken using HAADF STEM. The intensity of HAADF images is approximately proportional to $\mathrm{Z}^{2}$ (atomic number) times sample thickness and has little or no diffraction sensitivity. Correspondingly, gas bubbles have a darker contrast compared to the surrounding material, and denser materials have brighter contrast.

Comparison of the two samples irradiated to a lower dose with the sample irradiated to $2.9 \times 10^{17} \mathrm{ions} / \mathrm{cm}^{2}$ (Fig. 3), the same three distinct microstructure zones along the depth direction are observed. Moreover, the depth of the large bubble formation region is consistent among all three samples. The microstructures of the sample irradiated to $2.6 \times 10^{17}$ ions $/ \mathrm{cm}^{2}$ (Fig. 9 (a)) and the sample irradiated to $2.9 \times 10^{17}$ ions $/ \mathrm{cm}^{2}$ (Fig. 3) are especially alike because of their similar irradiation doses. However, the sample irradiated to $1.8 \times 10^{17} \mathrm{ions} / \mathrm{cm}^{2}$ exhibits noticeable differences in microstructure. The main differences are listed below.

1) The intergranular gas bubbles formed in this sample are less than $40 \mathrm{~nm}$, versus a few hundred nanometers in the other two samples.

2) The intergranular gas bubbles aggregate in dense groups near the original grain boundaries (shown in Fig. 9 (c)), in contrast to the spread of gas bubbles along the sub-micron grain boundaries in the other samples. Since all three samples were prepared from the same initial material, the difference in intergranular bubble morphology indicates that grain subdivision developed at a dose between $1.8 \times 10^{17}$ ions $/ \mathrm{cm}^{2}$ and $2.6 \times 10^{17}$ ions $/ \mathrm{cm}^{2}$.

3) Phase reversal was incomplete in the sample irradiated to $1.8 \times 10^{17} \mathrm{ions} / \mathrm{cm}^{2}$, which is evidenced by a significant amount of $\alpha$-U precipitates, for example the ones indicated with triangles in Fig. 9 (d), remaining in the region of large gas bubbles. The reason may be due to insufficient irradiation damage.

Examination of the sample irradiated to $1.8 \times 10^{17}$ ions $/ \mathrm{cm}^{2}$ provided evidence of the initiation of recrystallization. Fig. 10 (a), taken from Zone C in Fig. 9 (c), depicts the representative microstructure of an unirradiated cell boundary area. The observation of fine $\alpha$-U platelets clustered around cell boundaries, displayed in Fig. 10 (a), suggests that the $\alpha$-U particles are the Widmanstätten alpha structure, typically developed during quite short holding times at high temperatures [25]. On the contrary, no $\alpha-\mathrm{U}$ particle was found in the cell boundary area shown in Fig. 10 (b), but subgrain-like structures approximately a few hundred nanometers were observed, indicative of recrystallization. This area is located in Zone B before the Xe concentration peak, and the damage level 
is estimated to be $600-700$ dpa. Three subgrain-like structures were outlined and labeled in Fig. 10 (b) to show the possible recrystallized structure in the irradiated grain boundary area. Their boundaries have a slightly lighter contrast than their interiors. Because the sizes of these subgrain-like structures are smaller than the smallest SAD (Selected Area Diffraction) apertures in the two TEMs used in this study, it was not possible to determine the subgrain orientations. Nevertheless, the diffraction pattern taken on the area suggests that the subgrain-like structures have low-angle grain boundaries. The comparison of the microstructure presented in Figs. 10 (a) and (b) implies that irradiation-induced phase reversal is relevant to the initiation of recrystallization. In addition, the local Mo content might also impact the phase-reversal process: the lower the Mo content, the slower the phase reversion. Further detailed composition studies will be conducted to investigate whether irradiation-induced phase reversal gives rise to the initiation of recrystallization.

Aside from the $\alpha$-U platelets, some dark features were commonly observed at cell boundaries, for example the ones in the center of Figs. 10 (a) and (b). These features are most possibly $\mathrm{U}_{2} \mathrm{Mo}$, because $\alpha$-U phase is always accompanied with $\mathrm{U}_{2}$ Mo in the regions where $\gamma$-phase decomposes [25] [26]. Energy dispersive X-ray spectroscopy (EDS) analyses performed at the cell/grain boundaries were not definitive enough to confirm whether the features are Mo-rich precipitates or pits created during ion milling. Additional bright-field TEM observations will be carried out to identify the nature of those dark features.

\section{Discussion}

\subsection{Intra-granular gas bubble morphology}

It was known that intra-granular gas bubbles that formed in in-pile irradiation tend to self-organize in an fcc (face-center-cubic) lattice [17]-[19]. Miller et al. characterized the fission gas bubble superlattice in U-7Mo dispersion fuels irradiated in the ATR (Advanced Test Reactor) to various fission densities [27]. Their TEM observation showed that the fcc bubble superlattice, coherent with the body-center-cubic (bcc) U-Mo lattice structure, exists in all samples. However, bubble ordering was not observed in this study. The lack of ordering indicates that the irradiation conditions of this experiment were not suitable for gas bubble superlattice formation in terms of dose, damage rate, or temperature. Nevertheless, it is useful to compare irradiation conditions and bubble characteristics between neutron irradiation and ion irradiation in order to understand the formation mechanism of the gas bubble superlattice. 
Because fission gas behavior is the subject of interest, fission gas concentration is used as the criteria to establish a proper comparison. For this reason, the sample irradiated to $4.5 \times 10^{21}$ fissions $/ \mathrm{cm}^{3}$ in reactor from Ref. [27] was compared side by side with the sample irradiated to $2.9 \times 10^{17}$ ions $/ \mathrm{cm}^{2}$ with high-energy Xe ions, mainly because the two samples have similar Xe concentration. The major characteristics of the intra-granular gas bubble in these two samples are listed in Table 2, as well as the irradiation conditions. Because of the heterogeneity of gas atom distribution along sample depth in the ion irradiation, the averaged values over the bubble formation range ( 3 - $5.5 \mu \mathrm{m}$ from the surface) are presented in Table 2. It is shown that the intra-granular bubble size and density measured from the two samples are in a similar order. The ones formed under ion implantation have a slightly larger size and a lower density. The biggest difference uncovered is the ordered versus random intra-granular bubble morphology. Hence, more focus was placed on exploring the possible origins of the failure to form a bubble lattice in the samples irradiated in this study.

The phenomenon of bubble self-organization during energetic particle irradiation has been observed in many metal systems in the past [28]-[30]. However, the collected experimental observations show that the bubble ordering structure was only seen in a small number of samples within a narrow window of irradiation conditions (dose, dose rate, and temperature) [28]-[30]. A lot of irradiation studies which were performed under supposedly favorable conditions did not report the formation of a bubble lattice. Without a clear understanding of the formation mechanism of bubble superlattices, it is impossible to identify the irradiation parameter that leads to the inability to form a bubble lattice in this experiment. Nevertheless, the possible influences of each irradiation parameter on bubble morphology are explored. Firstly, it was observed that elevated temperature suppressed bubble lattice formation in Mo under ion irradiation [31]. The same situation may be extended to the current experiment. The ion irradiation temperature was $300-350^{\circ} \mathrm{C}$, much higher than the temperature of $130^{\circ} \mathrm{C}$ typical of in-pile irradiations. It is possible that bubble lattice formation is inhibited because of the relatively high temperature. On the other hand, the ion irradiation temperature is still within the reported temperature range for gas bubble lattice formation, which is $0.15 T_{m}$ to $0.35 T_{m}$ [30] ( $T_{m}$ is the melting temperature). Therefore, more systematic examinations are required in order to clarify the temperature effect. Secondly, the bubble lattice does not form when gas concentration is too high, evidenced by an earlier experiment of He implantation in Cu conducted by Johnson, Mazey and Diprose [28]. In this experiment bubble ordering was found at the depths shallower or deeper than the peak He concentration position, but not at the peak position. In the U-Mo alloy irradiated in reactor to $4.5 \times 10^{21}$ fissions $/ \mathrm{cm}^{3}$ [27], only part 
of bubble superlattice survived in the sample, and the majority was swept out by the formation of sub-grain boundaries. Van den Berghe et al. reported that the bubble superlattice has fully developed in U-7Mo at a fission density of $1.3 \times 10^{21}$ fissions $/ \mathrm{cm}^{3}$ [17]. At this fission density, the estimated gas concentration is $3.3 \times 10^{20} 1 / \mathrm{cm}^{3}$, which is much lower than that in this study $\left(8.7 \times 10^{20} 1 / \mathrm{cm}^{3}\right.$ for the lowest-dose sample). Hence, an upper limit of the irradiation dose for bubble lattice formation exists, and the doses obtained in this irradiation may exceed that limit. Finally, the dose rate may have a strong impact on the bubble lattice formation, although it has only a weak effect in void lattice formation [29]. Unlike void ordering, which occurs only at temperatures that allow sufficient vacancy migration, most bubble lattices were observed at room temperature [29], where the mobility of vacancies is mainly stimulated by radiation-enhanced diffusivity, and is proportional to fission rate/dose rate. Therefore, an appropriate dose rate may be important in forming ordered bubbles. In summary, the results observed from the current study are not sufficient to prove any one or a combination of the irradiation parameters inhibits bubble lattice formation.

\subsection{Radiation-induced phase reversion}

The stable form of a U-Mo alloy is a mixture of $\alpha$-phase uranium and $\mathrm{U}_{2} \mathrm{Mo}$ (often designated as the $\gamma^{\prime}$ phase) at low temperature, and a homogeneous single-phase $\gamma$-U solid solution above $570^{\circ} \mathrm{C}$ [26]. The cubic-structure (body centered cubic) $\gamma$ phase is desirable because of its high strength and stability. It was demonstrated that metastable $\gamma$ phase in U-Mo can be retained at room temperature by suitable quenching [26]. However, heating the metastable $\gamma$ phase over certain period will cause its transformation to $\alpha+\gamma^{\prime}$ [26]. The $\alpha$-U precipitates observed in this study resulted from thermally-induced $\gamma$-phase decomposition that occurred during fuel plate fabrication $\left(500^{\circ} \mathrm{C}\right.$ for $\sim 4$ hours). Under certain thermal conditions, the extent of decomposition is related to the Mo content in the U-Mo alloy, which was added in order to stabilize the $\gamma-\mathrm{U}$ phase. Since the cell boundary areas in a fuel particle normally have a lower Mo content than cell interiors [20][21], most of the $\alpha-U$ precipitates observed are located in the prior cell boundary areas.

It has been established that $\alpha$-transformed U-Mo can revert to the metastable $\gamma$-phase during neutron irradiation at low temperatures $\left(<200^{\circ} \mathrm{C}\right)$ [32][33]. The phase reversal was explained on the basis of homogenization induced by the high mobility of $U$ and Mo atoms, assuming a displacement [33] or a thermal spike [32] mechanism is operative. All three samples examined in this study show the occurrence of phase reversal, evidenced by the 
dissolution of $\alpha$-U particles in Zone B. This result confirms that high-energy heavy ion irradiation is equivalent to neutron irradiation in transforming decomposed $\alpha+\gamma^{\prime}$ back to the metastable $\gamma$-phase.

Neutron irradiation results also indicated that the diffusion coefficient of $U$ and Mo atoms within a displacement/thermal spike is proportional to damage rate [33] [34], the number of displacement/thermal spikes per unit volume per second. Therefore, the efficiency of phase reversal is dependent on the dpa (displacement per atom) rate, which is a function of dose rate and ion depth. Among the samples irradiated in this experiment, the dose rate of ATLAS2-L was the lowest. Correspondingly, the phase reversal process should be less effective in ATLAS2-L according to the models, which was confirmed by the observation that there were more residual $\alpha$ particles in ATLAS2-L than the other two samples. On the other hand, the fact that $\alpha$ particles only exist at the end of Zone B implies that the dpa rate across the entire ion path was adequate in revoking phase reversal except the tail positions of the ion distribution profile.

\subsection{Radiation-induced grain subdivision}

During in-pile irradiation, a microstructural change termed "recrystallization" was observed in U-Mo alloys irradiated to a fission density $>3 \times 10^{21}$ fissions $/ \mathrm{cm}^{3}$ [7][35][36], similar to the "rim effect" or "high burn-up structure (HBS)" found in the radial peripheral region of high burnup $\mathrm{UO}_{2}$ fuel rods [37]. SEM images of the fracture surface of irradiated U-Mo fuels show that new small grains started to nucleate at prior cell boundaries [35], accompanied by the formation of a large number of intergranular bubbles. With the increase of fission density, front lines of the sub-micron-grain clusters propagated into the interior of the original grain, and eventually the fine grains occupied the entire material. It was believed that fission gas swelling in U-Mo fuel is promoted in this process [7] because of the combined effects of shortened gas diffusion distances to grain boundaries, increased grain boundary area per unit volume, and accelerated intergranular bubble growth rate [38].

Concerning the driving mechanism of recrystallization, there is no experimental evidence or clear theoretical description available currently. Rest presented a theoretical model of recrystallization in U-Mo fuel to describe the process from initiation to completion [38]-[40]. The model attributes recrystallization to the lattice distortion caused by the formation of a cellular dislocation network during irradiation. This model was originally proposed by Rest and Hofman to explain the recrystallization phenomena in $\mathrm{UO}_{2}$ [41] and modified to be applicable to U-Mo by adjusting the applicable materials properties. 
The apparent microstructural changes after heavy ion bombardment, exhibited in Fig. 3, Fig. 9 (a), and Fig. 10 provide new perspectives on the underlying mechanisms of recrystallization in U-Mo. The split of the original large cells into small grains delineated with large bubbles in Figs. 3 and 9(a) gives clear evidence of irradiation-induced recrystallization. In Fig. 10, the concurrency of the dissolution of $\alpha-U$ particles and the formation of subgrain-like structures on prior grain boundaries suggests the possible connection between the two phenomena. This rationality comes from the well-known fact that substantial lattice distortions accompany uranium phase transformation [42]. It is expected that stresses stored in the lattice increased significantly when the lattice atoms were rearranged from one crystal structure to another during irradiation-induced phase reversal. The recrystallization process can therefore occur to release the accumulated stresses through forming a new set of defect-free small grains. This postulation can successfully explain that original grain boundaries are the preferential initiation sites of the recrystallization process, which was not clarified in earlier models. Furthermore, since Fig. 10 (b) was taken in a region outside of the Xe bubble formation region, it is suggested that the formation of subgrains in U-Mo does not require the presence of a high concentration of fission products, as suggested by some models used to explain rim effect in $\mathrm{UO}_{2}$ [37]. Additional characterization and irradiation experiments need to be performed targeted at understanding the progress of recrystallization.

\section{Conclusion}

Three distinct zones were observed along the ion traveling direction in U-7Mo in all three U-7Mo/Al dispersion fuel samples irradiated with $84 \mathrm{MeV}$ Xe ions at $350^{\circ} \mathrm{C}$ to various doses. The microstructural characteristics of each microstructural zone are described as follows.

A. The surface layer is the U-7Mo reacted with $\mathrm{Al}$ sputtered from the nearby $\mathrm{Al}$ matrix. This layer is about $1 \mu \mathrm{m}$ thick and composed of nano-crystalline $(\mathrm{U}, \mathrm{Mo}) \mathrm{Al}_{\mathrm{x}}$;

B. The second layer is U-Mo implanted with Xe ions. High-density small bubbles formed within U-7Mo grains starting from $\sim 3 \mu \mathrm{m}$ from the surface. The intra-granular bubbles have an average size of $4.7 \pm 1.3 \mathrm{~nm}$ and a density of $3.1 \times 10^{17} 1 / \mathrm{cm}^{3}$ in the sample irradiated to $2.9 \times 10^{17} \mathrm{ions} / \mathrm{cm}^{2}$. No particular spatial ordering of these nano-size bubbles was detected. Linearly arranged large gas bubbles up to hundreds of nanometers in size formed at the peak position of the Xe distribution profile estimated with SRIM. The morphology of these large bubbles resembles the recrystallized area in in-pile irradiated fuels. 
C. The material in the third layer is beyond the Xe deposition range and, therefore, retains the un-irradiated U-7Mo microstructure. The typical microstructure of this region is a $\gamma-\mathrm{U}$ grain interior surrounded by a band of dense $\alpha$ U particles induced by thermal decomposition of the low-Mo-content cell/grain boundary areas during fuel plate fabrication.

The experimental observations acquired in this study and their comparison to the damaged U-Mo microstructures formed in in-pile irradiations point to several important findings that helps understand in-pile behavior of the U-Mo fuel. These findings are: (i) Although the irradiation conditions in this experiment cannot yield a gas bubble superlattice as shown in in-pile irradiated fuels, the intra-granular bubble size and density are not far from those measured from a gas bubble superlattice. (ii) The $\alpha$-U particles within the Xe-implanted region were reverted to $\gamma$-U phase by irradiation. This result is the same as what was observed in in-pile irradiation and can be explained on the basis of composition homogenization caused by the high atom mobility within displacement or thermal spikes. (iii) Irradiation-induced recrystallization, observed in the high burnup zones of fuel plates irradiated in reactors, occurred under high-energy, high-dose Xe ion irradiation. The high lattice stresses built up during the irradiation-induced phase reversal is probably the driving force for the small grain formation at cell boundaries. Further detailed composition and grain orientation study will be conducted to verify the connection between irradiation-induced phase reversal and recrystallization in U-Mo alloys.

\section{Acknowledgements}

This work was supported by the U.S. Department of Energy, National Nuclear Security Administration (NNSA), Office of Material Management and Minimization (NA-23) Reactor Conversion Program. Part of the electron microscopy was accomplished at Argonne National Laboratory at the IVEM-Tandem Facility, a U.S. Department of Energy Facility funded by the DOE Office of Nuclear Energy, operated under Contract No. DE-AC02-06CH11357 by UChicago Argonne, LLC. Some of the TEM and all STEM characterization work were supported by the U.S. Department of Energy, Office of Nuclear Energy under DOE Idaho Operations Office Contract DE-AC0705ID14517, as part of Advanced Test Reactor National Scientific User Facility (ATR NSUF) experiments. 


\section{References}

[1] J.L. Snelgrove, G.L. Hofman, M.K. Meyer, C.L. Trybus, T.C. Wiencek, Nucl. Eng. Des. 178 (1997) 119-126.

[2] G.L. Hofman, M.K. Meyer, J.M. Park, "Observations on the irradiation behavior of U-Mo Alloy dispersion fuel," Proceedings of the $23^{\text {rd }}$ International Meeting on Reduced Enrichment for Research and Test Reactors (RERTR), Las Vegas, Nevada, October 1-6 (2000).

[3] S. Van Den Berghe, P. Lemoine, Nucl. Eng. Technol. 46 (2014) 125-146.

[4] A. Leenaers, S. Van den Berghe, C. Detavernier, J. Nucl. Mater. 440 (2013) 220-228.

[5] D.M. Wachs, A.B. Robinson, F.J. Rice, N.C. Kraft, S.C. Taylor, M. Lillo, N. Woolstenhulme, G.A. Roth, J. Nucl. Mater. 476 (2016) 270-292.

[6] Y.S. Kim, G.L. Hofman, J. Nucl. Mater. 419 (2011) 291-301.

[7] Y.S. Kim, G.L. Hofman, J.S. Cheon, J. Nucl. Mater. 436 (2013) 14-22.

[8] N. Wieschalla, A. Bergmaier, P. Böni, K. Böning, G. Dollinger, R. Großmann, W. Petry, A. Röhrmoser, J. Schneider, J. Nucl. Mater. 357 (2006) 191-197.

[9] H. Palancher, N. Wieschalla, P. Martin, R. Tucoulou, C. Sabathier, W. Petry, J. Berar, C. Valot, S. Dubois, J. Nucl. Mater. 385 (2009) 449-455.

[10] H-Y. Chiang, T. Zweifel, H. Palancher, A. Bonnin, L. Beck, P. Weiser, M. Döblinger, C. Sabathier, R. Jungwirth, W. Petry, J. Nucl. Mater. 440 (2013) 117-123.

[11]B. Ye, S. Bhattacharya, K. Mo, D. Yun, W. Mohamed, M. Pellin, J. Fortner, Y.S. Kim, G.L. Hofman, A.M. Yacout, T. Wiencek, S. Van den Berghe, A. Leenaers, J. Nucl. Mater. 464 (2015) 236-244.

[12] G.L. Hofman, M.K. Meyer, J.M. Park, "Observations on the irradiation behavior of U-Mo Alloy dispersion fuel," Proceedings of the $23^{\text {rd }}$ International Meeting on Reduced Enrichment for Research and Test Reactors (RERTR), Las Vegas, Nevada, October 1-6 (2000).

[13] K. Nogita, K. Hayashi, K. Une, K. Fukuda, J. Nucl. Mater. 273 (1999) 302-309.

[14] J. Ziegler, SRIM, 2010, http://www.SRIM.org.

[15]Rasband, W.S., ImageJ, U. S. National Institutes of Health, Bethesda, Maryland, USA, http://imagej.nih.gov/ij/, 1997-2016.

[16] M.H. Loretto, R.E. Smallman, Defect analysis in electron microscopy, Chapman and Hall, 1975.

[17] S. Van den Berghe, W. Van Renterghem, A. Leenaers, J. Nucl. Mater. 375 (2008) 340-346.

[18] J. Gan, D.D. Keiser Jr., D.M. Wachs, A.B. Robinson, B.D. Miller, T.R. Allen, J. Nucl. Mater. 396 (2010) 234239.

[19] J. Gan, B.D. Miller, D.D. Keiser Jr., A.B. Robinson, J.W. Madden, P.G. Medvedev, D.M. Wachs, J. Nucl. Mater. 454 (2014) 434-445.

[20] K.H. Kim, D.B. Lee, C.K. Kim, G.L. Hofman, K.W. Park, J. Nucl. Mater. 245 (1997) 179-184.

[21] K.H. Kim, J.M. Park, C.K. Kim, G.L. Hofman, M.K. Meyer, Nucl. Eng. Des. 211 (2002) 229-235.

[22] J.M. Park, H.J. Ryu, K.H. Kim, D.B. Lee, Y.S. Lee, J.S. Lee, B.S. Seong, C.K. Kim, M. Cornen, J. Nucl. Mater. 397 (2010) 27-30.

[23] A. Bonnin, J. Wright, R. Toucoulou, H. Palancher, Appl. Phys. Lett. 105 (2014) 084103.

[24] G. Champion, R. Belin, H. Palancher, X. Iltis, H. Rouquette, M. Pasturel, V. Demange, P. Castany, V. Dorcet, O. Tougait, Powder Technol. 255 (2014) 29-35.

[25] P.E. Repas, R.H. Goodenow, R.F. Hehemann, Trans. ASM 57 (1964) 150-163.

[26] G. Beghi, Gamma phase uranium-molybdenum fuel alloys, European Atomic Energy Community, 1968.

[27] B.D. Miller, J. Gan, D.D. Keiser Jr., A.B. Robinson, J.F. Jue, J.W. Madden, P.G. Medvedev, J. Nucl. Mater. 458 (2015) 115-121.

[28] P.B. Johnson, Gas Bubble Lattice in Metals, in S.E. Donnelly and J.H. Evans (Ed.), Fundamental Aspects of Inert Gases in Solids (167-184). Plenum Press, New York, 1991.

[29]K. Krishan, Radiat. Eff. 66 (1982) 121-155.

[30] N.M. Ghoniem, D. Walgraef, S.J. Zinkle, J. Comp. Aided Mater. Des. 8 (2002) 1-38.

[31]D.J. Mazey, B.L. Eyre, J.H. Evans, S.K. Erents, G.M. McCraken, J. Nucl. Mater. 64 (1977) 145.

[32] S.T. Konobeevsky, K.P. Dubrovin, B.M. Levitsky, L.D. Panteleev, N.F. Pravdyuk, Proceeding of International Conference on the peaceful uses of atomic energy, 232-249 (1958).

[33] M.L. Bleiberg, L.J. Jones, B. Lustman, J. Appl. Phys. 27 (1956) 1270-1283.

[34] M.L. Bleiberg, J. Nucl. Mater. 2 (1959) 182-190.

[35] A. Leenaers, W. Van Renterghem, S. Van den Berghe, J. Nucl. Mater. 476 (2016) 218-230. 
[36] G.L. Hofman, Y.S. Kim, Nucl. Eng. Tech. 37 (2005) 299-308.

[37] V.V. Rondinella, T. Wiss, Mater. Today 13 (2010) 24-32.

[38] J. Rest, J. Nucl. Mater. 346 (2005) 226-232.

[39] J. Rest, J. Nucl. Mater. 349 (2006) 150-159.

[40] J. Rest, J. Nucl. Mater. 326 (2004) 175-184.

[41] J. Rest, G.L. Hofman, J. Nucl. Mater. 277 (2000) 231-238.

[42] H.H. Klepfer, P. Chiotti, Characteristics of the solid state transformation in uranium, Ames laboratory ISC technical reports, paper 163, 1957. 


\section{List of tables}

Table 1. Irradiation parameters of the ion-irradiated U-7Mo samples analyzed with TEM

Table 2. Comparison of irradiation conditions and intra-granular bubble characters between neutron- and ionirradiated U-Mo fuels. 
Table 1. Irradiation parameters of the ion-irradiated U-7Mo samples analyzed with TEM

\begin{tabular}{cccccc}
\hline Sample ID & $\begin{array}{c}\text { Average dose rate } \\
\left.\text { (ions } / \mathrm{cm}^{2} / \mathrm{s}\right)\end{array}$ & $\begin{array}{c}\text { Final dose } \\
\left(\text { ions } / \mathrm{cm}^{2}\right)\end{array}$ & Peak dpa & $\begin{array}{c}\text { Location on the } \\
\text { irradiation stage [11] }\end{array}$ & $\begin{array}{c}\text { Coated with } \\
\text { ZrN }\end{array}$ \\
\hline ATLAS2-J & $9.2 \times 10^{11}$ & $2.9 \times 10^{17}$ & 1206 & Center & Yes \\
ATLAS2-O & $8.2 \times 10^{11}$ & $2.6 \times 10^{17}$ & 1081 & Ring 1 & Yes \\
ATLAS2-L & $5.7 \times 10^{11}$ & $1.8 \times 10^{17}$ & 748 & Ring 3 & No \\
\hline
\end{tabular}

Table 2. Comparison of irradiation conditions and intra-granular bubble characteristics between neutron- and ionirradiated U-Mo fuels.

\begin{tabular}{lll}
\hline & ATR reactor irradiation [27] & Heavy ion irradiation \\
\hline Irradiation source & neutron & $84 \mathrm{MeV} \mathrm{Xe} \mathrm{ion}$ \\
Irradiation temperature $\left({ }^{\circ} \mathrm{C}\right)$ & 130 & $300-350$ \\
Fission density or dose & $4.5 \times 10^{21} \mathrm{fissions} / \mathrm{cm}^{3}$ & $2.9 \times 10^{17} \mathrm{ions} / \mathrm{cm}^{2}$ \\
Damage rate & $3.8 \times 10^{14} \mathrm{fissions} / \mathrm{cm}^{3} / \mathrm{s}$ & $1.1 \times 10^{-3} \mathrm{dpa} / \mathrm{s}$ \\
Xe concentration $\left(1 / \mathrm{cm}^{3}\right)$ & $1.1 \times 10^{21}$ & $1.4 \times 10^{21}$ \\
Intra-granular bubble morphology & partial fcc lattice & random \\
Intra-granular bubble diameter $(\mathrm{nm})$ & $3.5 \pm 0.4$ & $4.7 \pm 1.3$ \\
Intra-granular bubble density $\left(1 / \mathrm{cm}^{3}\right)$ & $1.4 \times 10^{18}$ & $3.1 \times 10^{17}$ \\
\hline
\end{tabular}




\section{List of figures}

Figure 1. Outline of sample preparation procedure before irradiation.

Figure 2. SRIM-calculated 84-MeV Xe ion distribution and damage profile in U-7Mo.

Figure 3. A montage of TEM images of the U-7Mo sample irradiated with $84 \mathrm{MeV}$ Xe ions to a dose of $2.9 \times 10^{17}$ ions $/ \mathrm{cm}^{2}$.

Figure 4. The magnified view of the "A" area marked in Fig. 3, showing the microstructure of the (U,Mo)Al $\mathrm{x}_{\mathrm{x}}$ layer.

Figure 5. The magnified views of the "B" area marked in Fig. 3 taken at the (a) overfocus and (b) underfocus conditions, showing the high density bubbles inside fuel grains.

Figure 6. Intra-granular bubble density and average diameter as a function of distance from surface in the bubble formation region in the sample irradiated with Xe to a dose of $2.9 \times 10^{17} \mathrm{ions} / \mathrm{cm}^{2}$.

Figure 7. (a) Bright-field TEM image, (b) selected area diffraction pattern, and (c) dark-field image of utilizing precipitate reflection indicated in SAD in (b) taken on the " $\mathrm{C}$ " area marked in Fig. 3, showing the existence of second-phase precipitates beyond the ion deposition range.

Figure 8. (a) Bright-field TEM micrograph of an unirradiated U-7Mo sample along with (b) the SAD pattern showing the existence of $\alpha-\mathrm{U}$ precipitates at grain boundaries.

Figure 9. HAADF-STEM micrographs of U-Mo samples irradiated with Xe to doses of $2.6 \times 10^{17}$ ions $/ \mathrm{cm}^{2}$ ((a) overview and (b) magnified view of the boxed region in (a)) and $1.8 \times 10^{17} \mathrm{ions} / \mathrm{cm}^{2}$ ((c) overview and (d) magnified view of the boxed region in (c)) respectively.

Figure 10. STEM micrographs showing representative microstructure of (a) unirradiated U-Mo grain boundary area and (b) irradiated grain boundary area in the sample irradiated to $1.8 \times 10^{17} \mathrm{ions} / \mathrm{cm}^{2}$. 


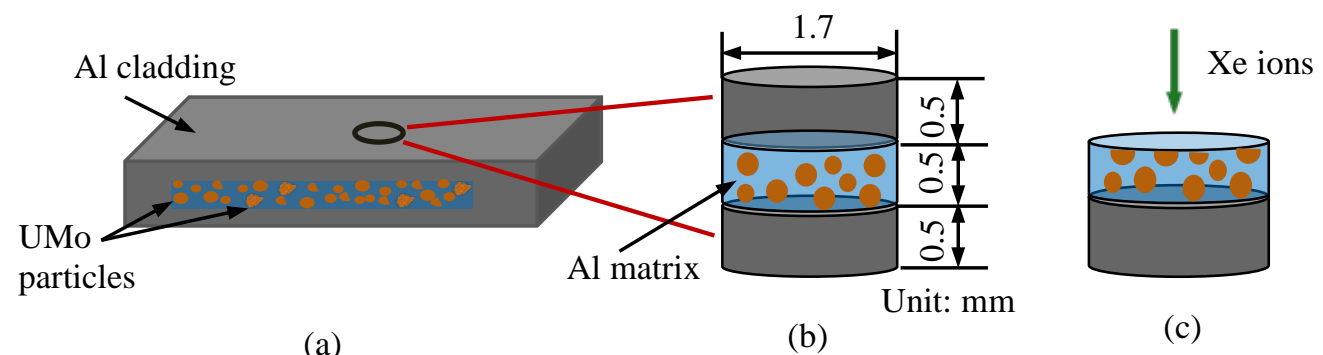

Figure 1. Outline of sample preparation procedure before irradiation.

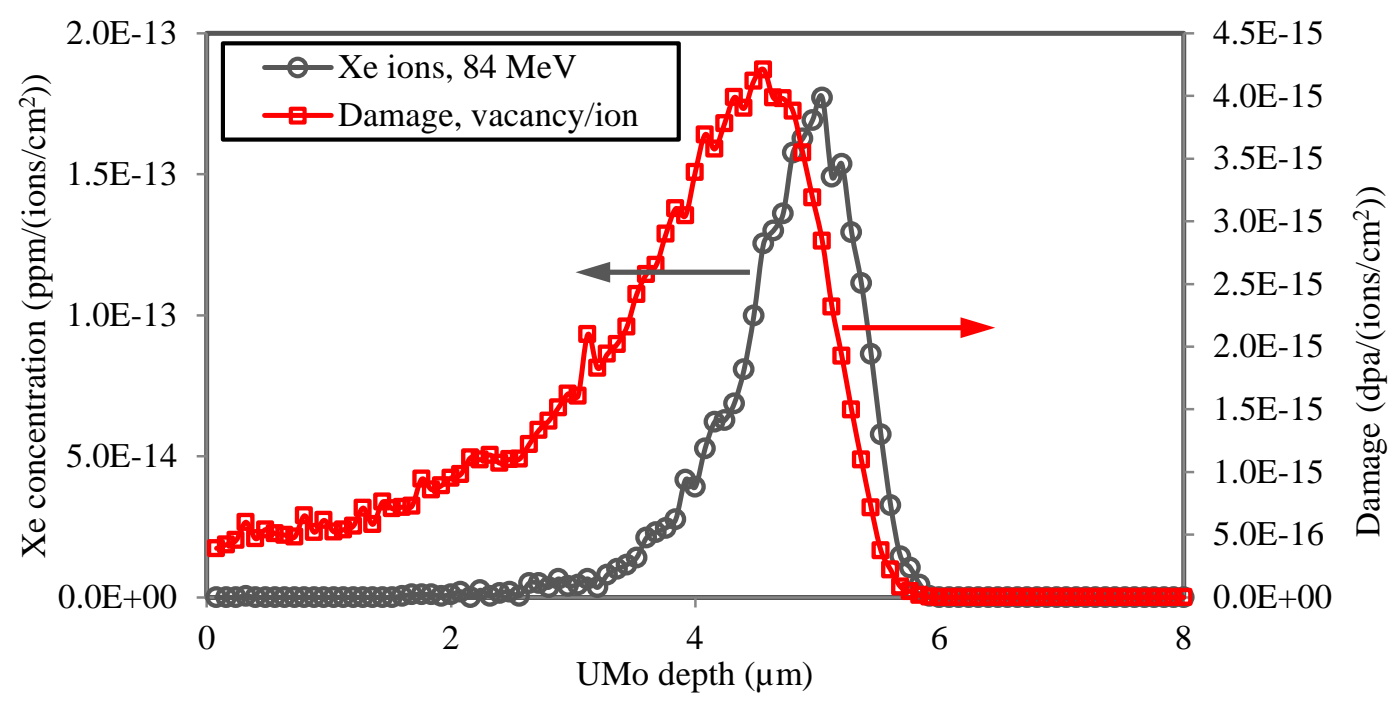

Figure 2. SRIM-calculated 84-MeV Xe ion distribution and damage profile in U-7Mo. 


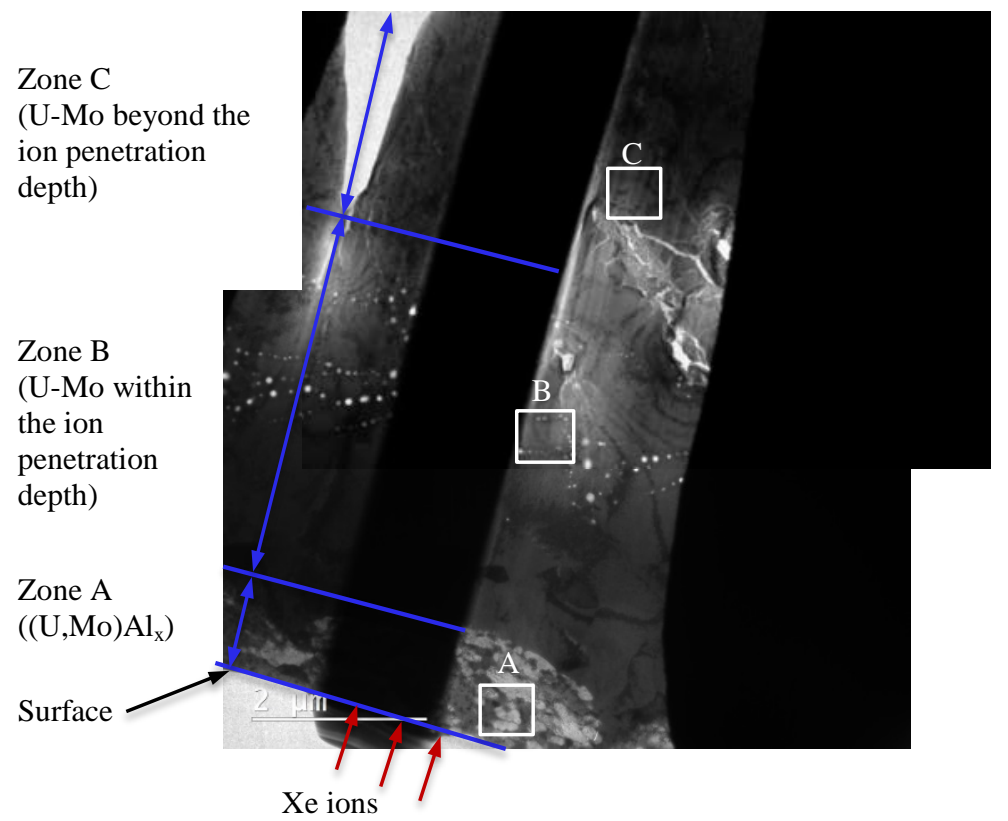

Figure 3. A montage of TEM images of the U-7Mo sample irradiated with $84 \mathrm{MeV}$ Xe ions to a dose of $2.9 \times 10^{17}$ ions $/ \mathrm{cm}^{2}$.

(a)

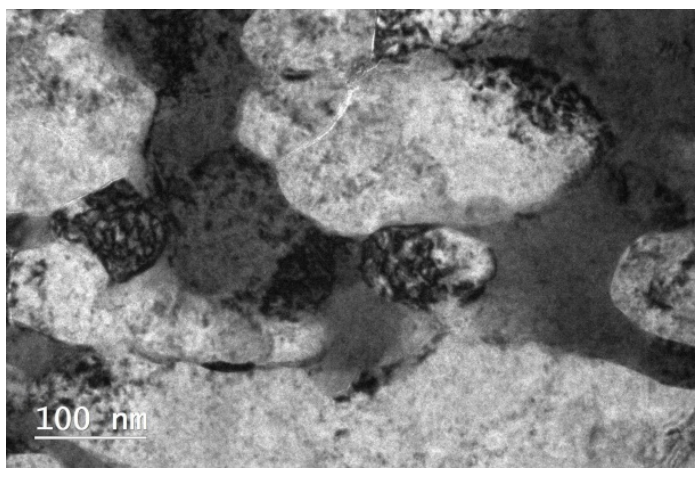

(b)

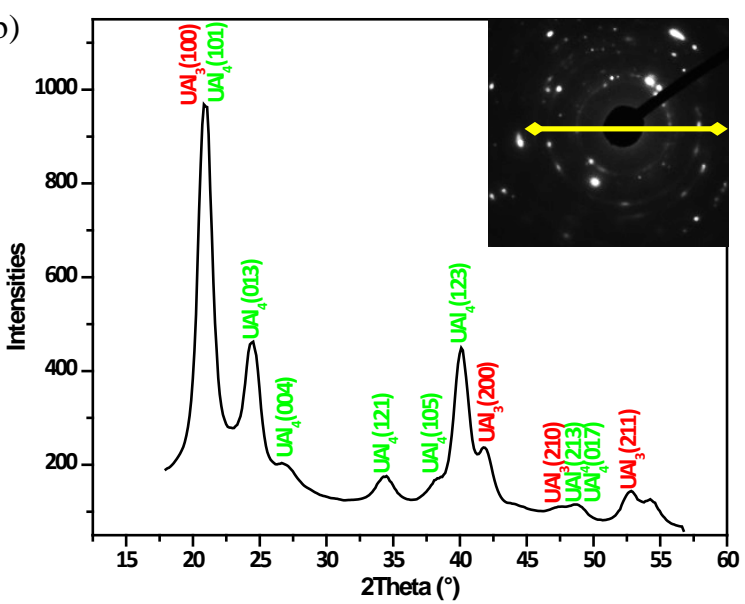

Figure 4. The microstructure of the (U,Mo)Al $\mathrm{l}_{\mathrm{x}}$ layer: (a) the magnified view of the "A" area marked in Fig. 3; (b) indexing of the intensity variation of diffraction rings across the diffraction pattern (inset) shows that its major phases are $\mathrm{UAl}_{3}$ and $\mathrm{UAl}_{4}$. 

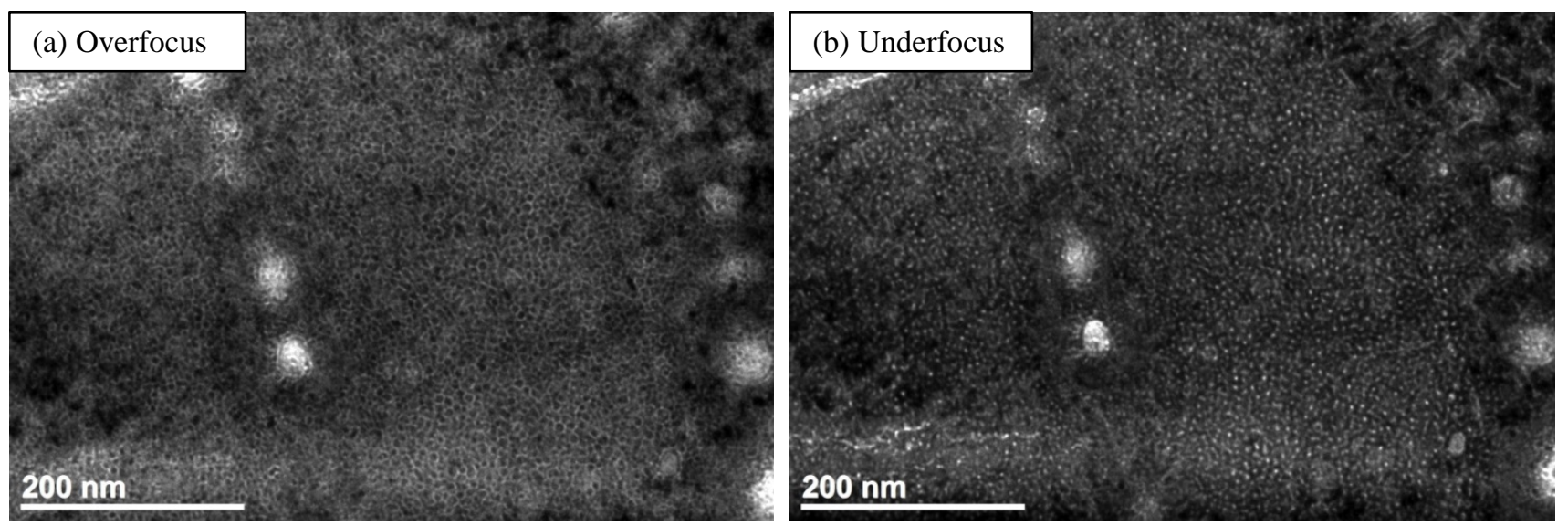

Figure 5. The magnified views of the "B" area marked in Fig. 3 taken at the (a) overfocus and (b) underfocus conditions in the bright-field TEM mode, showing the high density bubbles inside fuel grains.

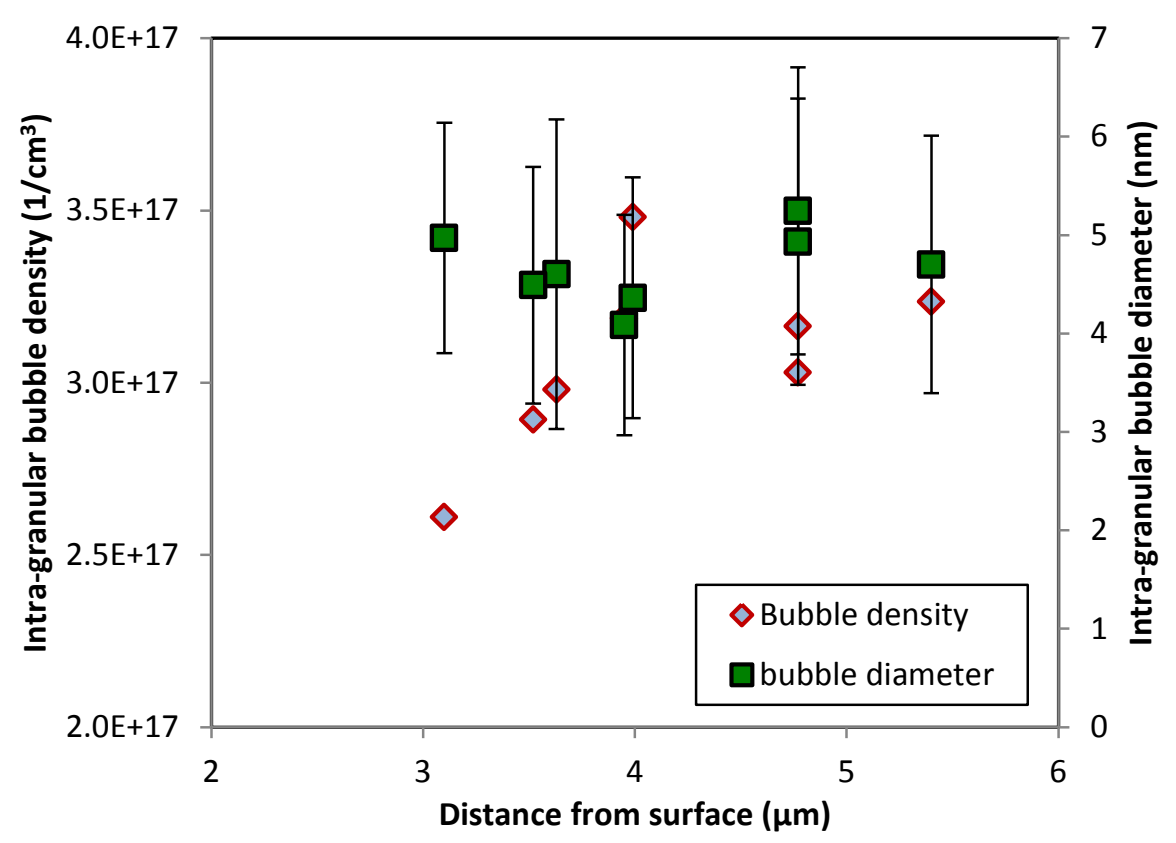

Figure 6. Intra-granular bubble density and average diameter as a function of distance from surface in the bubble formation region in the sample irradiated with Xe to a dose of $2.9 \times 10^{17}$ ions $/ \mathrm{cm}^{2}$. 


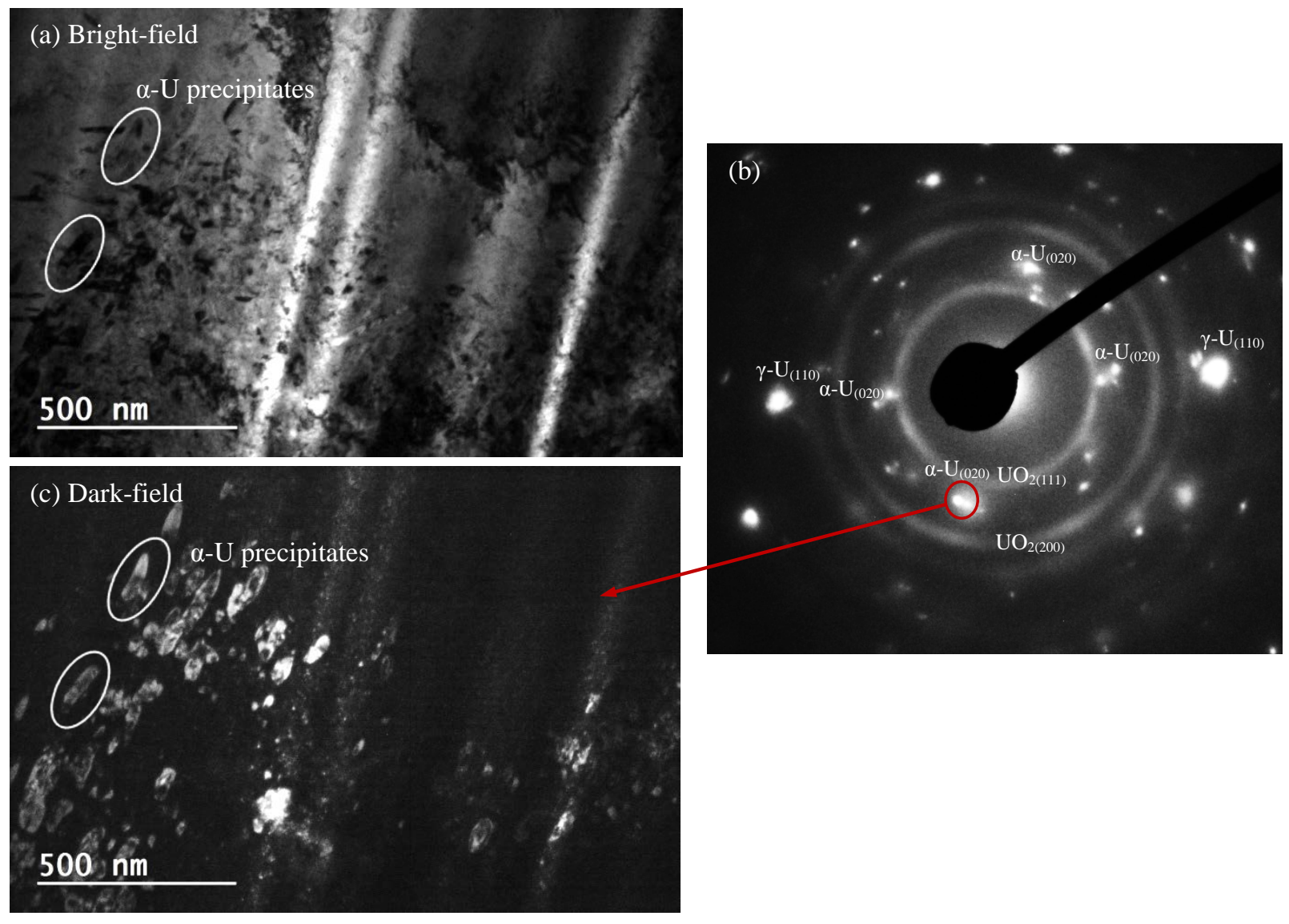

Figure 7. (a) Bright-field TEM image, (b) selected area diffraction pattern, and (c) dark-field image of utilizing precipitate reflection indicated in SAD in (b) taken on the " $\mathrm{C}$ " area marked in Fig. 3, showing the existence of second-phase precipitates beyond the ion deposition range. 

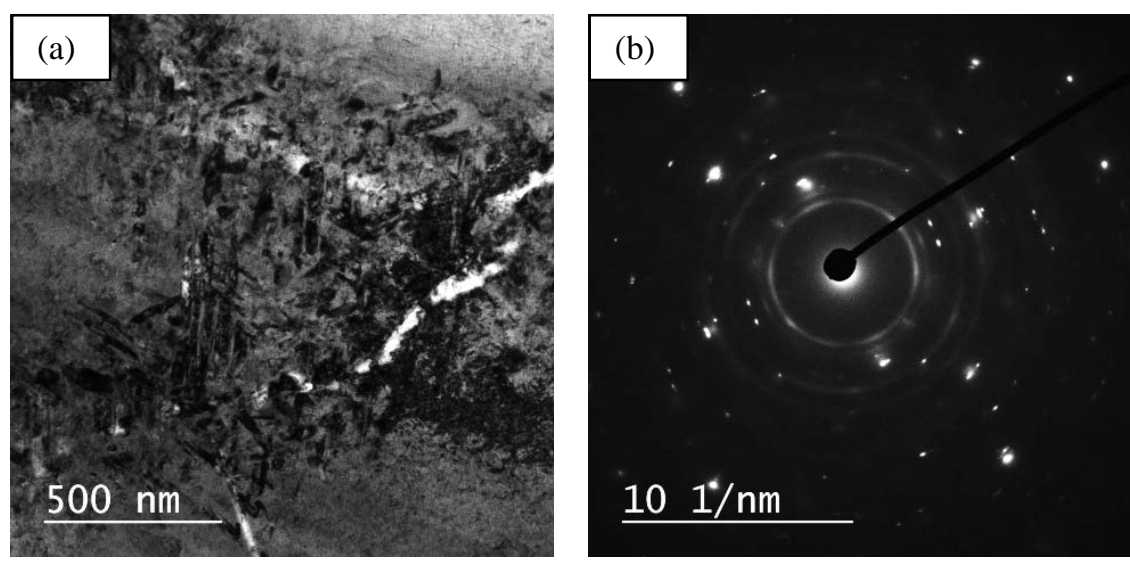

Figure 8. (a) Bright-field TEM micrograph of an unirradiated U-7Mo sample along with (b) the SAD pattern showing the existence of $\alpha-\mathrm{U}$ precipitates at grain boundaries. 

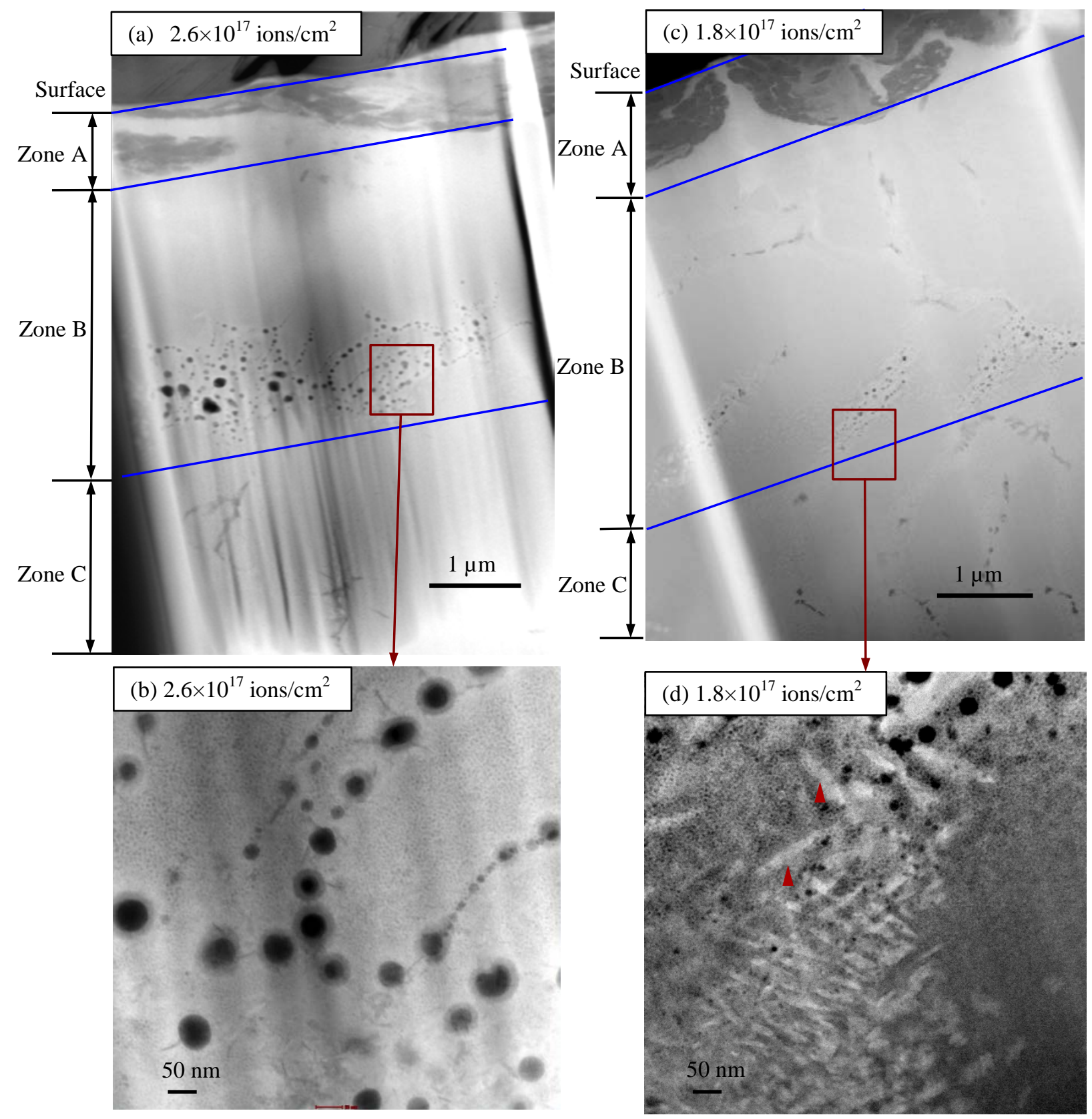

Figure 9. HAADF-STEM micrographs of U-Mo samples irradiated with Xe to doses of $2.6 \times 10^{17} \mathrm{ions} / \mathrm{cm}^{2}$ ((a) overview and (b) magnified view of the boxed region in (a)) and $1.8 \times 10^{17} \mathrm{ions} / \mathrm{cm}^{2}$ ((c) overview and (d) magnified view of the boxed region in (c)) respectively. Arrows in (d) indicate the $\alpha$-U particles. 

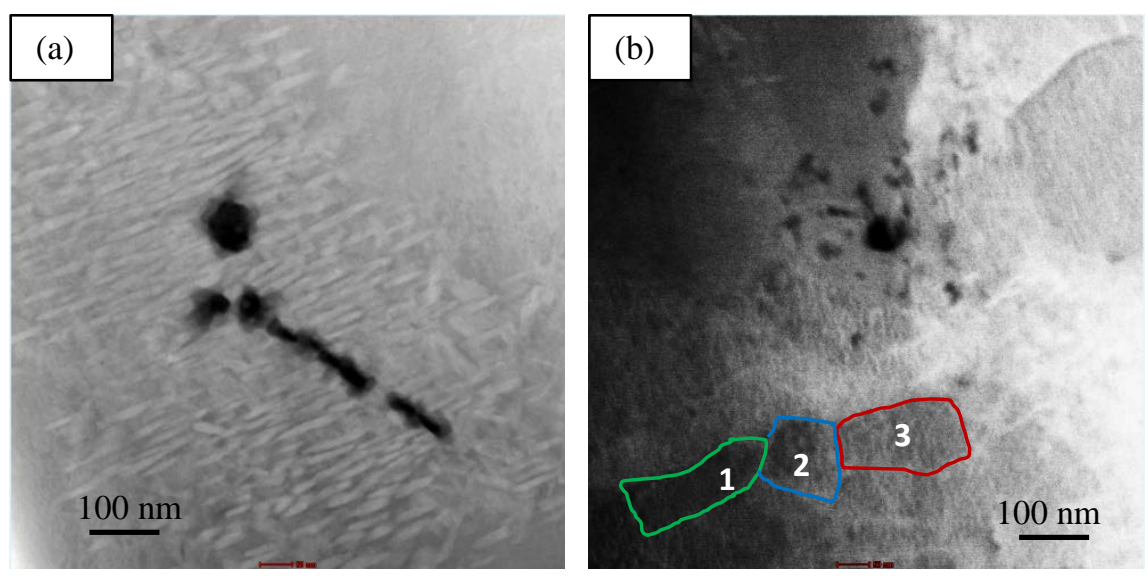

Figure 10. STEM micrographs showing representative microstructure of (a) unirradiated U-Mo grain boundary area and (b) irradiated grain boundary area in the sample irradiated to $1.8 \times 10^{17}$ ions $/ \mathrm{cm}^{2} ; 3$ subgrain-like structures were outlined to show the possible recrystallized structure. 